\title{
Reduced Dopamine Transporter Functioning Induces High-Reward Risk-Preference Consistent with Bipolar Disorder
}

\author{
Jordy van Enkhuizen ',2, Brook L Henry', Arpi Minassian', William Perry', Morgane Milienne-Petiot ${ }^{1,2}$, \\ Kerin K Higa', Mark A Geyer ${ }^{1,3}$ and Jared W Young*,1,3 \\ 'Department of Psychiatry, University of California San Diego, La Jolla, CA, USA; ²Division of Pharmacology, Utrecht Institute for Pharmaceutical \\ Sciences, Utrecht University, Utrecht, The Netherlands; ${ }^{3}$ Research Service, VA San Diego Healthcare System, San Diego, CA, USA
}

\begin{abstract}
Individuals with bipolar disorder (BD) exhibit deleterious decision making, negatively impacting their lives. Such aberrant decision making can be quantified using the lowa Gambling Task (IGT), which requires choosing between advantageous and disadvantageous options based on different reward/punishment schedules. The mechanisms underlying this behavioral deficit are unknown, but may include the reduced dopamine transporter (DAT) functioning reported in BD patients. Using both human and mouse IGTs, we tested whether reduced DAT functioning would recreate patterns of deficient decision making of BD patients. We assessed the IGT performance of $16 \mathrm{BD}$ subjects (7 female) and 17 healthy control $(\mathrm{HC})$ subjects ( 12 female). We recorded standard IGT performance measures and novel post-reward and post-punishment decision-making strategies. We characterized a novel single-session mouse IGT using C57BL/6) mice $(n=44)$. The BD and HC IGT performances were compared with the effects of chronic (genetic knockdown (KD; $n=31)$ and wild-type $(n=28)$ mice) and acute (C57BL/6) mice $(n=89)$ treated with the DAT inhibitor GBR I2909) reductions of DAT functioning in mice performing this novel IGT. BD patients exhibited impaired decision making compared with HC subjects. Both the good-performing DAT KD and GBR I2909-treated mice exhibited poor decision making in the mouse IGT. The deficit of each population was driven by highreward sensitivity. The single-session mouse IGT measures dynamic risk-based decision making similar to humans. Chronic and acute reductions of DAT functioning in mice impaired decision-making consistent with poor IGT performance of BD patients. Hyperdopaminergia caused by reduced DAT may impact poor decision making in BD patients, which should be confirmed in future studies. Neuropsychopharmacology (2014) 39, 3II2-3I22; doi:I0.1038/npp.2014.170; published online 6 August 2014
\end{abstract}

\section{INTRODUCTION}

Several psychiatric disorders are associated with impaired decision making (Christodoulou et al, 2006; Fond et al, 2013; Mantyla et al, 2012), deleteriously impacting the patients' quality of life (Jollant et al, 2007). Neural networks contributing to decision making have been identified (Floresco et al, 2008; Lin et al, 2008). Clarification of the mechanism(s) underlying decision making in psychiatric patients requires delineation for targeted therapeutic development. The Iowa Gambling Task (IGT) utilizes highyield/high-risk versus low-yield/low-risk options to measure decision making with real-world translational validity in one test-session (Bechara et al, 1994). Patients with bipolar disorder (BD) exhibit poor IGT performance (Adida et al, 2011; Ibanez et al, 2012). Moreover, a diagnosis-specific performance profile can be discerned. Manic BD patients

*Correspondence: Dr JW Young, Department of Psychiatry, University of California San Diego, 9500 Gilman Drive MC 0804, La Jolla, CA 92093-0804, USA, Tel: + I 619543 3582, Fax: + I 6197359205 ,

E-mail: jaredyoung@ucsd.edu

Received 6 March 20I4; revised 25 June 20I4; accepted 28 June 20 I4; accepted article preview online 9 July 2014 are hypersensitive to rewards (Cassidy et al, 1998). Schizophrenia patients exhibit disrupted contingency learning (Brambilla et al, 2012), whereas depressed patients are more sensitive to punishment (Adida et al, 2011; Must et al, 2013). Collectively, these findings support differing mechanisms underlying poor decision making in these disorders.

Model animals for these impairments are required for treatment development (Insel, 2007). Based on the human IGT, animal analogs have been created (de Visser et al, 2011). In the rodent IGT, animals are presented with four options with different reward/punishment probabilities and magnitudes. Consistent with the human IGT, two options offer small rewards and little punishment (safe/advantageous choices), whereas the other two options offer larger rewards and more punishment (risky/disadvantageous choices). The effects of dopaminergic, serotonergic, and noradrenergic manipulations have been investigated in rats and mice using a rodent IGT, in which learning was acquired and examined across multiple test sessions (Baarendse et al, 2013; van Enkhuizen et al, 2013b; Zeeb et al, 2009; Zeeb et al, 2013). These studies revealed various neurotransmitter involvements on already learned decisionmaking processes. In contrast to these tasks, however, the human IGT examines dynamic decision-making during 
learning in a single session, limiting the translational validity of multiple session rodent IGT studies (de Visser et al, 2011). In support of this assertion, similar experimental manipulations in multiple- $v s$ single-session rodent IGTs produced different results. Orbitofrontal cortical lesions exaggerated deficits of rats in within-session (Rivalan et al, 2011) but not learned (Zeeb and Winstanley, 2011) rat decision-making in single- $v s$ multiple session IGTs, respectively. In the single-session rodent IGT, good, intermediate, and poor decision-making rodents can be identified based on their risk-learning performance (Rivalan et al, 2009). Interindividual differences are also observed among healthy humans performing the IGT (Bechara and Damasio, 2002a; Weller et al, 2010). Assessment of mechanisms that may contribute to impaired IGT performance in BD could therefore be conducted in mice by using a single-session IGT that enables direct comparisons with human IGT findings.

Although mechanisms underlying BD symptoms remain poorly defined, increasing evidence suggests that elevated dopamine (DA) levels most likely have a key role (Manji et al, 2003; Vawter et al, 2000). One key mechanism of DA homeostasis is its reuptake from the synaptic cleft into presynaptic nerve terminals by the DA transporter (DAT). Because several studies support an important role of DA in regulating risk-based decision making in rodents (Floresco et al, 2008; St Onge and Floresco, 2009), altered DAT functioning may contribute to abnormal decision making in individuals with BD. Supporting this assumption, polymorphisms in the DAT gene have been linked with BD (Greenwood et al, 2006; Pinsonneault et al, 2011), likely lowering functional DAT levels (Horschitz et al, 2005). Reduced striatal DAT levels are seen in unmedicated BD patients (Anand et al, 2011) and postmortem tissue (Rao et al, 2012). Thus, the relationship between reduced DAT functioning and its contribution to impaired risk-based decision-making in $\mathrm{BD}$ patients may be important, but remains undetermined.

Previously, we reported that mice with reduced DAT functioning mimicked abnormal behaviors of $\mathrm{BD}$ mania patients (Perry et al, 2009). Mice that are hyperdopaminergic via genetic DAT knockdown (KD; Zhuang et al, 2001) or pharmacological DAT inhibition (GBR12909 treatment) exhibited this profile. To determine whether reduced DAT functioning could impair decision making under risk, we compared single-session IGT performance of patients with $\mathrm{BD}$ and mice with chronic (DAT KD) and acute (GBR12909) reductions of DAT functioning. A single-session IGT was used for consistency with the human IGT and to measure dynamic changes in decision making after rewards or punishments. We predicted that: (1) decision making would be impaired in $\mathrm{BD}$ patients; (2) subpopulations of mice would be identifiable in the mouse IGT based on risk learning; and (3) both chronic and acute reductions of DAT functioning would impair IGT performance similar to deficits observed in BD.

\section{MATERIALS AND METHODS}

\section{Participants}

Sixteen participants (18-55 years old) who met SCID (Structured Clinical Interview for DSM-IV) criteria for BD were recruited from inpatient and outpatient psychiatric clinics located at the University of California San Diego (UCSD) Medical Center. Nine participants met criteria for a current manic episode (Young Mania Rating Scale score $\geqslant 20$ ) and seven were classified as hypomanic (Young Mania Rating Scale score $=12-15$; Tohen et al, 2002). The majority of patients were taking mood stabilizers and/or atypical antipsychotics with the most common medication being valproate and lithium or risperidone, respectively. Seventeen healthy control (HC) participants who had never met SCID criteria for any Axis I psychiatric disorder and did not have first-degree relatives with $\mathrm{BD}$ were recruited from advertisements in the San Diego community. BD and HC groups were matched for age, gender, education, and ethnicity, and had equivalent premorbid IQ as assessed by the Peabody Picture Vocabulary Test (Dunn and Dunn, 1997; Table 1). Participants were excluded for: (1) current alcohol or substance dependence; (2) a history of neurological conditions, head trauma, or seizures; (3) treatment with electroconvulsive therapy; (4) stroke or myocardial infarction; and (5) a positive result for cocaine, amphetamine, or phencyclidine on a urine toxicology Rapid Drug screen (Pharmatic Inc., San Diego, CA) administered during the test session. All subjects provided written informed consent to the current protocol approved by the UCSD Institutional Review Board known as the Human Research Protections Program.

\section{Human IGT}

Participants were administered a computerized version of the IGT where individuals were required to select from four decks of cards (A, B, C, and D; Bechara et al, 1994). This IGT is a decision-making measure included in the National

Table I Demographic and Clinical Characteristics of BD Patients and HC Subjects

\begin{tabular}{|c|c|c|c|}
\hline Parameter & $\mathrm{HC}(n=17)$ & $\operatorname{BD}(n=16)$ & $\begin{array}{c}\text { Group } \\
\text { differences }\end{array}$ \\
\hline Age (years) & $33.9 \pm 3.0$ & $33.8 \pm 2.8$ & NS \\
\hline Gender (male/female) & $5 \mathrm{M}, 12 \mathrm{~F}$ & $9 \mathrm{M}, 7 \mathrm{~F}$ & NS \\
\hline Education (years) & $14.8 \pm 0.6$ & $13.9 \pm 0.6$ & NS \\
\hline Ethnicity (\% Caucasian) & $47 \%$ & $75 \%$ & NS \\
\hline Peabody Picture Vocabulary Test & $104.7 \pm 2.7$ & $96.9 \pm 4.2$ & NS \\
\hline BD age of onset (years) & & $23.0 \pm 1.8$ & \\
\hline BD duration of illness (years) & & $10.1 \pm 1.9$ & \\
\hline Number of BD hospitalizations & & $2.7 \pm 0.5$ & \\
\hline YMRS score & $0.9 \pm 0.8$ & $22.3 \pm 2.3^{* * * * *}$ & $\mathrm{BD}>\mathrm{HC}$ \\
\hline HDRS score & $0.9 \pm 0.4$ & 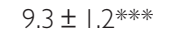 & $\mathrm{BD}>\mathrm{HC}$ \\
\hline \multicolumn{4}{|l|}{ Medication } \\
\hline Antipsychotic alone & & 3 & \\
\hline Mood stabilizer alone & & I & \\
\hline Antipsychotic + mood stabilizer & & 9 & \\
\hline Other medications & & 0 & \\
\hline Not medicated & & 3 & \\
\hline
\end{tabular}

Abbreviations: BD, bipolar disorder; HC, healthy control; HDRS, Hamilton Depression Rating Scale; YMRS, Young Mania Rating Scale. **** $p<0.001$ 
Institute on Drug Abuse PhenX Toolkit (http://phenxtoolkit.org- 31 January 2014, Version 5.7) as part of the National Institute on Drug Abuse-endorsed initiative to promote data harmonization across studies. After selecting a card, a theoretical amount of money was displayed on the screen. Decks A or B resulted in high monetary gains, but also high unpredictable penalties (disadvantageous). Decks C and D paid smaller amounts of money but incurred smaller losses (advantageous; Supplementary Table 1). The task included 100 trials and participants were informed that the goal was to avoid losing money and win as much money as possible (Figure 1). Decision making was measured using a net score calculated by subtracting disadvantageous choices $(A+B)$ from the advantageous choices $(C+D)$.

\section{Mice}

Male C57BL/6J $(n=133)$, DAT KD $(n=31)$, and wild-type (WT) littermate $(n=28)$ mice were used throughout the experiments. DAT heterozygous breeders backcrossed onto a C57BL/6J background for $>10$ generations were sent to UCSD from the University of Chicago. All mice were 3-5 months old at the time of testing and weighed between 21 and $34 \mathrm{~g}$. All animals were group housed (maximum four/ cage) and maintained in a temperature-controlled vivarium $\left(21 \pm 1{ }^{\circ} \mathrm{C}\right)$ on a reversed day-night cycle (lights on at 1900 hours, off at 0700 hours). All mice had ad libitum access to water and were food-restricted at $85 \%$ of their free-feeding weight during the periods of testing (during the dark phase of the day-night cycle between 0800 hours and 1800 hours). All procedures were approved by the UCSD Institutional Animal Care and Use Committee. The UCSD animal facility meets all federal and state requirements for animal care.

\section{Drugs}

GBR12909 dihydrochloride was purchased from Sigma Aldrich (St Louis, MO, USA) and dissolved in saline after heating $\left(45^{\circ} \mathrm{C}, 60 \mathrm{~min}\right.$; van Enkhuizen et al, 2013a; Young et al, 2010). GBR12909 was injected intraperitoneally with a volume of $10 \mathrm{ml} / \mathrm{kg}$, immediately before testing. Free-base drug weight was used in drug calculations.

\section{Mouse IGT}

A single-session IGT was developed for mice based on the original task designed by Rivalan et al (2009). Sixteen five-hole operant chambers were used for the IGT (Supplementary Methods) to provide four illuminated options consistent with the task in rats. In short, mice had $10 \mathrm{~s}$ to holepoke in one of four illuminated holes. Mice were rewarded with strawberry milkshake or punished with a time-out period depending on the reward schedule (Figure 1). Consistent with the original rat task, two options delivered large rewards or long time-out penalties (disadvantageous), whereas the other two options delivered smaller rewards or shorter time-out penalties (advantageous). The reward and punishment probabilities used here were consistent with those of Rivalan et al (2009). The high punishment durations used here for mice were lower, however, 66 and $132 \mathrm{~s}$ compared with 222 and $444 \mathrm{~s}$ because of the punishment sensitivity observed in mice. Even with this reduced duration, advantageous choices correlated most strongly with avoiding punishment as opposed to gaining rewards (Supplementary Figure 8), unlike rats (Rivalan et al, 2009). Decision making was measured as \%advantageous choices and several other measures were recorded and presented (Table 2).

\section{Characterization of the Single-Session Mouse IGT}

After stabilization of responding on a simple fixed-ratio schedule (Supplementary Materials and Methods), singlesession performance of C57BL/6J mice $(n=44)$ was assessed in the IGT.
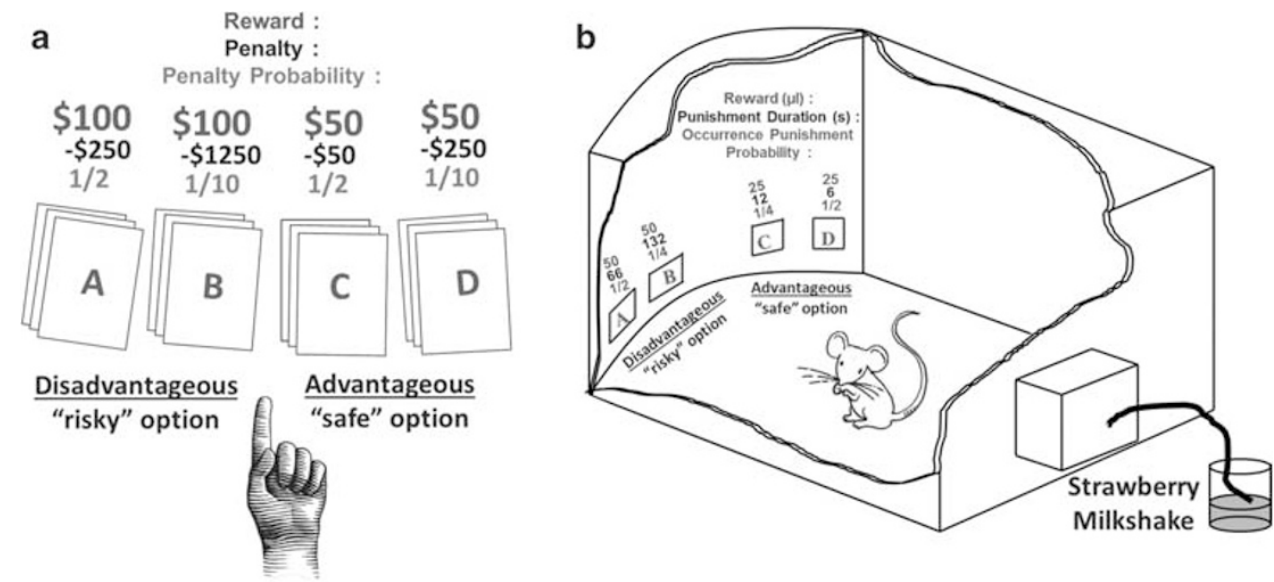

Figure I Illustrative comparison between the human lowa Gambling Task (IGT) and the single-session mouse IGT. (a) Human subjects sequentially selected one card among four available decks for 100 trials and were instructed to try to win as much money as possible. (b) The IGT for mice was developed based on the original work in rats by Rivalan et al. After operant training, mice were tested in the single-session I-h long IGT. After a 5-s delay, the mouse could holepoke among the illuminated holes (A-D) for a I0-s period. For the human and mouse paradigms, the selected card or hole determined the given reward value (money for humans, strawberry milkshake for mice; in red), or punishment value (monetary loss for humans, flashing cue light for mice; in black), according to the associated probability (in blue). Based on preliminary data, lower punishment durations were used for mice compared with rats. In both IGTs, two small reward/low punishment options (C and D) were ultimately advantageous, whereas the other two high reward/high punishment options ( $A$ and $B$ ) were disadvantageous. 
Table 2 Description of the Behavioral Measures Used in the Single-Session Mouse lowa Gambling Task

\begin{tabular}{ll}
\hline Measures & Description \\
\hline \% Advantageous choices: & Advantageous response options $[(C+D) /$ total $(A+B+C+D)] \times 100$ \\
\% Disadvantageous choices: & Disadvantageous response options $[(A+B) /$ total $(A+B+C+D)] \times 100$ \\
(P) Safe-stay & Probability of choosing advantageous options after being rewarded from advantageous options \\
$(p)$ Risky-stay & Probability of choosing disadvantageous options after being rewarded from disadvantageous options \\
(P) Safe-shift & Probability of choosing disadvantageous options after being punished from advantageous options \\
(P) Risky-shift & Probability of choosing advantageous options after being punished from disadvantageous options \\
Omissions (\%): & Failure to respond in any hole during the light stimulus duration of I2s (motivation) \\
Premature responses: & Response in any cue hole during the 5-s inter-trial interval preceding illumination of the cue array (motor impulsivity) \\
Mean choice latency $(\mathrm{s}):$ & The latency to holepoke in one of the four holes (reaction time) \\
\hline
\end{tabular}

Effects of Chronic Reductions of DAT Functioning on IGT Performance

DAT KD $(n=31)$ and WT littermates $(n=28)$ were trained to holepoke. After stabilization of responding, the IGT performance of these mice were assessed and compared.

\section{Effects of Acute Reductions of DAT Functioning on IGT Performance}

After stabilization of responding, C57BL/6J mice $(n=89)$ naïve to the IGT received saline $(n=29)$, GBR12909 at 9 $(n=30)$ or $16 \mathrm{mg} / \mathrm{kg}(n=30$; van Enkhuizen et al, 2013b; Young et al, 2010) and were challenged in the IGT.

\section{Quantifying Individual Differences in Learning Performance}

Based on previous observations (Rivalan et al, 2009), we examined the performance of individual mice and identified three different subpopulations. The total trials of each group were split into three trial periods based on their total trials completed. These subpopulations were quantified by subtracting $\%$ advantageous choices of trial period 1 from $\%$ advantageous choices of trial period 3. Good, intermediate, and poor decision makers were stratified as (1) $>0.5$, (2) between 0.5 and -0.5 , and $(3)<0.5$ standard deviations from the mean, respectively. This stratification was made for each genotype or drug treatment group separately.

\section{Post-Reward/Punishment Decision-Making: Win-Stay/ Lose-Shift Strategies}

The likelihood of a subject repeatedly choosing a card/ stimulus following a reward from the advantageous options (safe-stay) and disadvantageous options (risky-stay) was compared with their likelihood of selecting a different choice following punishment from the advantageous (safeshift) and disadvantageous options (risky-shift; Supplementary Methods).

\section{Statistical Analyses}

Human and mouse choices were analyzed over three equal blocks of trials. Human IGT net score was analyzed using analysis of variance with group $(\mathrm{BD}, \mathrm{HC})$ as a between-subject factor and trial block as a within-subject factor. Animal choices were analyzed using analyses of variance with trial period as a within-subject factor, and genotype, drug, and group as between-subject factors. Group was determined by quantification of learning as described above. Subjects with $\leqslant 10$ completed trials per trial period were excluded from analysis. The \% advantageous choice preference was compared with chance (50\%) using a one-sample $t$-test. Tukey post hoc analyses of statistically significant main or interaction effects were performed where applicable and Cohen's $d$ effect sizes were calculated. Where appropriate, planned comparison paired $t$-tests were conducted between groups. The $\alpha$ level was set at 0.05. All analyses were performed using SPSS (19.0, Chicago, IL, USA).

\section{RESULTS}

\section{Human IGT Performance}

There was a significant difference in decision-making ability between $\mathrm{HC}$ and $\mathrm{BD}$ subjects $(\mathrm{F}(1,31)=6.7, p<0.05$; Figure 2a), indicating that $\mathrm{BD}$ subjects made less advantageous choices compared with HC subjects $(p<0.05$; effect size $[d]=0.91)$. Net score increased significantly over three trial blocks in the $\mathrm{HC}$ group $(\mathrm{F}(2,32)=3.5, p<0.05$; Figure $2 \mathrm{~b})$ with only a trend in the $\mathrm{BD}$ subjects $(\mathrm{F}(2,30)=$ 2.9, $p=0.070$ ). Traditional IGT analyses over five trial blocks indicated that net score increased significantly over time in both the $\mathrm{HC}(\mathrm{F}(4,64)=3.3, p<0.05)$ and $\mathrm{BD}$ subjects $(\mathrm{F}(4,60)=3.1, p<0.05$; Supplementary Figure 1$)$. Interestingly, despite the majority of HC subjects increasing their net score over time, some individuals did not, whereas others decreased advantageous preference over time (Figure 2e).

\section{Win-Stay/Lose-Shift Strategies in BD Subjects}

All subjects increased safe-stays over time $(\mathrm{F}(2,62)=5.3$, $p<0.05$; Supplementary Figure $2 \mathrm{~A}$ ), but the BD subjects tended to make less safe-stays compared with HCs $(\mathrm{F}(1,31)=4.0, p=0.054$; Figure $2 \mathrm{c})$. No significant effects were observed for risky-stays (Supplementary Figure 2B). $\mathrm{BD}$ subjects made more safe-shifts compared with HCs $(\mathrm{F}(1,31)=5.0, p<0.05$; Figure $2 \mathrm{~d})$, but there were no effects for risky-shifts (Supplementary Figure 2D). Hence, BD 
a

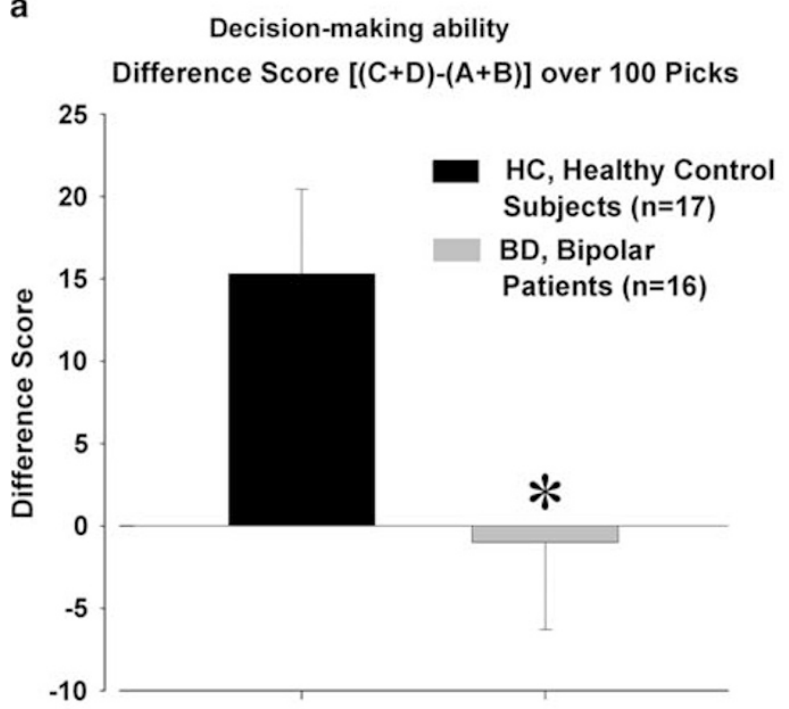

b

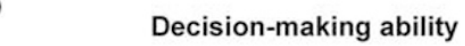

Difference Score $[(C+D)-(A+B)]$ over time

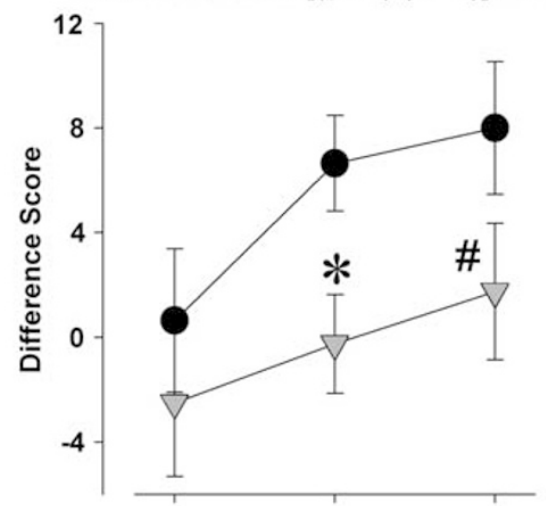

c
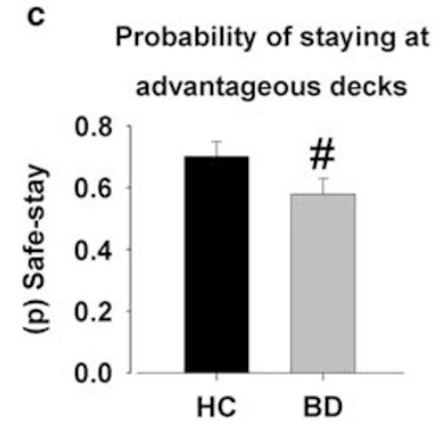

\section{d}

Probability of shifting from

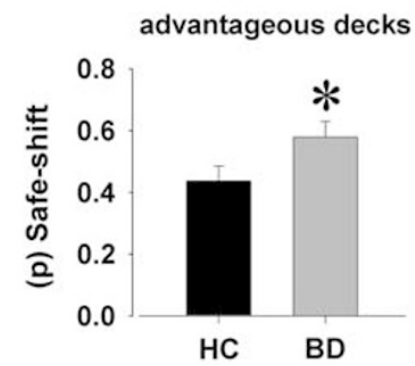

e

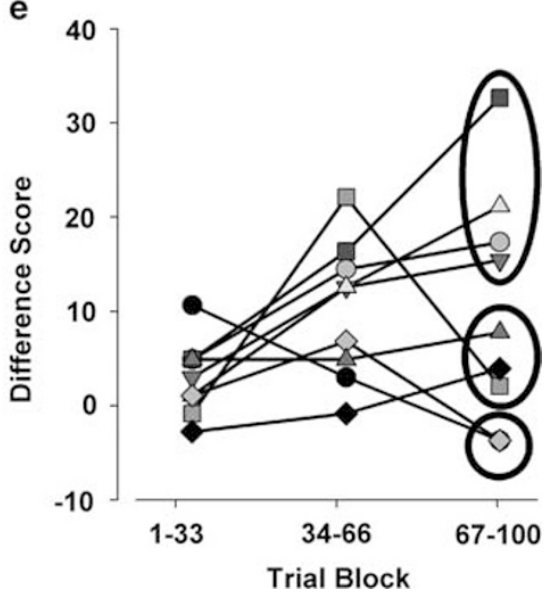

Figure 2 lowa Gambling Task (IGT) performance of bipolar disorder (BD) and healthy control (HC) subjects. (a) Over 100 card picks, the BD group had a significantly lower net difference score compared with HC subjects. (b) When analyzed over three trial blocks, BD subjects performed poorly compared with $\mathrm{HC}$ subjects in blocks two and three. (c) Compared with HC subjects, BD subjects tended to make fewer low-reward safe-stays. (d) Compared with $\mathrm{HC}$ subjects, BD subjects switched more often to the high-reward decks directly after losing at the low-reward decks (safe-shifts). (e) Inter-individual differences of a subset of $\mathrm{HC}$ subjects $(n=9)$ are displayed, indicating that the majority increases their net score over time, whereas others remain the same or decrease their net score over time. Data are presented as the mean \pm SEM, ${ }^{*} p<0.05$ and ${ }^{\#} p<0.1$ when compared with $\mathrm{HC}$ subjects.

subjects were less likely to stay at the safe low-reward options but as likely to stay at the risky high-reward options.

\section{Inter-Individual Differences of C57BL/6J Mice in a Single-Session IGT}

Mice were grouped into good (32\%), intermediate (48\%), or poor $(20 \%)$ decision makers based on their IGT learning performance (see Methods).

Advantageous choices. A group $\times$ trial period interaction $(\mathrm{F}(4,82)=19.1, p<0.001$; Figure $3 \mathrm{a})$ indicated that good decision makers increased advantageous choices $(\mathrm{F}(2,26)=$ $12.0, p<0.01)$, whereas poor decision makers decreased advantageous choices $(\mathrm{F}(2,16)=16.9, p<0.001)$ over time.

Other behavioral measures. Independent of group, mice reacted faster over time $(\mathrm{F}(2,82)=4.8, p<0.05)$, whereas omissions and premature responses did not $\operatorname{differ}(\mathrm{F}<1$, NS) over time (Table 3).

\section{Win-Stay/Lose-Shift Strategies of C57BL/6J Mice}

Good decision makers exhibited more safe-stays over time $(\mathrm{F}(2,26)=3.8, p<0.05$; Figure $3 \mathrm{~b})$ and compared with poor decision makers at the end of the session $(p<0.01)$. Poor decision makers, however, tended to exhibit more riskystays over time $(\mathrm{F}(2,16)=3.1, p=0.074$; Supplementary Figure $3 \mathrm{~A}$ ) and compared with good decision makers at the end of the session $(p<0.05)$. Poor decision makers exhibited more safe-shifts compared with good decision makers in trial period $3(p<0.01$; Figure $3 \mathrm{c})$, while they made less risky-shifts over time $(\mathrm{F}(2,16)=5.1, p<0.05$; Supplementary Figure 3B).

\section{IGT Performance of DAT WT and KD Mice}

A main group effect $(\mathrm{F}(2,53)=4.4, p<0.05)$, trial period $\times$ group interaction $(F(4,106)=30.2, p<0.001)$, and trends 

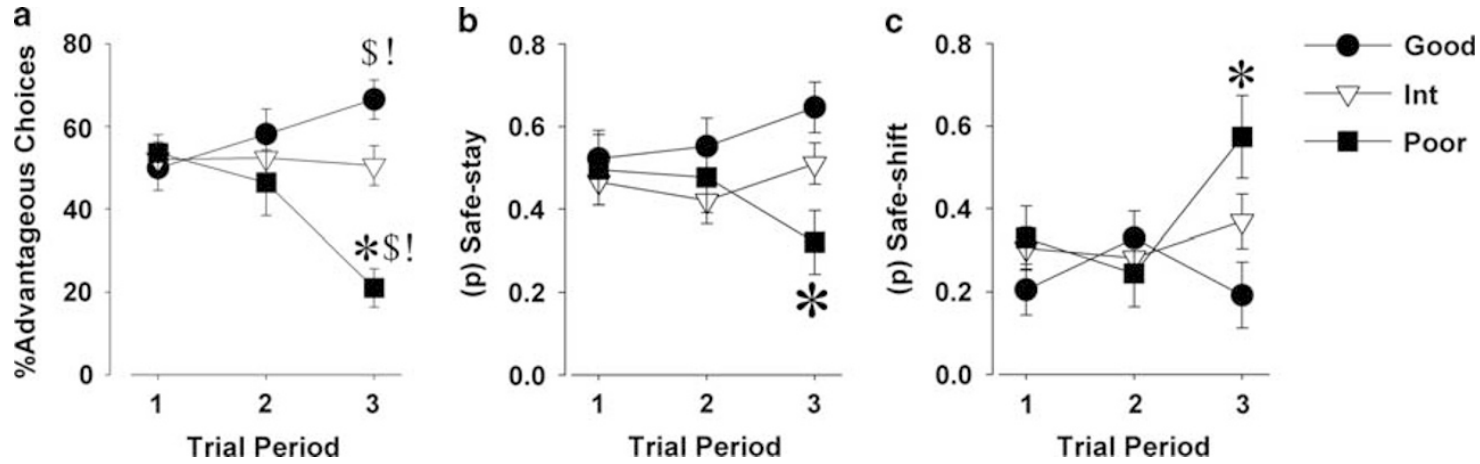

Figure 3 lowa Gambling Task (IGT) performance of C57BL/6) mice identified as good, intermediate, and poor decision makers. (a) Good decision makers chose advantageous options, whereas poor decision makers preferred disadvantageous options as the session progressed. (b) Good decision makers made more safe-stays over time, and compared with poor decision makers at the end of the session. (c) By the final trial period, poor decision makers made more safe-shifts compared with good decision makers. Data are presented as the mean \pm SEM, ${ }^{*} p<0.05$ when compared with good decision makers, ${ }^{\$} p<0.05$ when compared with intermediate decision makers, and $! p<0.05$ when compared with chance.

Table 3 Motivational/Motor Impulsivity Measures of Mice in the lowa Gambling Task

\begin{tabular}{llcccrrr}
\hline Measure & Group & C57 & WT & KD & Saline & GBR 9 & GBR I6 \\
\hline Mean choice latency (s) & Good & $2.86 \pm 0.24$ & $4.09 \pm 0.24$ & $3.37 \pm 0.20^{\mathrm{a}}$ & $4.31 \pm 0.36$ & $3.72 \pm 0.30$ & $4.91 \pm 0.30$ \\
& Interm & $3.11 \pm 0.20$ & $4.07 \pm 0.21$ & $3.11 \pm 0.24^{\mathrm{a}}$ & $4.88 \pm 0.26$ & $4.04 \pm 0.32^{\mathrm{a}}$ & $4.25 \pm 0.27^{\mathrm{b}}$ \\
& Poor & $3.30 \pm 0.30$ & $4.70 \pm 0.41$ & $3.37 \pm 0.34^{\mathrm{a}}$ & $4.57 \pm 0.42$ & $4.59 \pm 0.29$ & $4.45 \pm 0.33$ \\
Omissions (\%) & Good & $7.60 \pm 3.05$ & $17.21 \pm 2.82$ & $6.31 \pm 2.30^{\mathrm{a}}$ & $18.75 \pm 4.31$ & $7.65 \pm 3.60^{\mathrm{a}}$ & $24.17 \pm 3.60$ \\
& Interm & $10.79 \pm 2.49$ & $14.23 \pm 1.80$ & $7.62 \pm 2.14^{\mathrm{a}}$ & $20.72 \pm 3.16$ & $17.84 \pm 3.80$ & $23.68 \pm 3.29$ \\
& Poor & $14.93 \pm 3.80$ & $17.77 \pm 2.47$ & $7.26 \pm 2.02^{\mathrm{a}}$ & $23.84 \pm 5.10$ & $21.29 \pm 3.44$ & $17.10 \pm 4.03$ \\
Premature responses & Good & $5.21 \pm 1.82$ & $18.13 \pm 10.83$ & $47.00 \pm 8.84^{\mathrm{b}}$ & $1.05 \pm 1.55$ & $2.23 \pm 1.30$ & $2.90 \pm 1.30$ \\
& Interm & $9.10 \pm 1.48$ & $17.79 \pm 6.01$ & $47.70 \pm 7.11^{\mathrm{a}}$ & $3.69 \pm 1.14$ & $1.52 \pm 1.37$ & $3.44 \pm 1.19$ \\
& Poor & $6.67 \pm 2.27$ & $10.83 \pm 8.63$ & $32.33 \pm 7.05^{\mathrm{b}}$ & $2.40 \pm 1.84$ & $1.42 \pm 1.24$ & $4.50 \pm 1.45$ \\
\hline
\end{tabular}

Abbreviations: Interm, intermediate; KD, knockdown; WT, wild type.

${ }^{a} p<0.05$.

${ }_{p} p<0.1$ when compared with WT/saline.

toward trial period $\times$ genotype $(\mathrm{F}(2,106)=3.1, p=0.058)$ and trial period $\times$ genotype $\times$ group interactions $(\mathrm{F}(4,106)=$ 2.3, $p=0.078$ ) were observed. Similar to $\mathrm{C} 57 \mathrm{BL} / 6 \mathrm{~J}$ mice, good decision makers increased and poor decision makers decreased advantageous choices over time.

Good decision makers. Both $\mathrm{WT}$ and $\mathrm{KD}$ mice made increased advantageous choices over time $(\mathrm{F}(2,36)=27.6$, $p<0.001)$. A trial period $\times$ genotype interaction $(\mathrm{F}(2,36)=$ 5.0, $p<0.05$ ) indicated that KD mice made significantly less advantageous choices than WT mice during trial period $2(p<0.05$; effect size $[d]=1.22$; Figure $4 a)$. Importantly, WT mice performed above chance in trial periods 2 and 3 $(p<0.05)$, whereas KD mice did not differ from chance. No genotype differences were observed in either the intermediate or poor decision makers (Supplementary Figure 4A and $\mathrm{B})$.

Other behavioral measures. KD mice exhibited fewer omissions $(\mathrm{F}(1,53)=24.1, p<0.001)$, increased premature responses $(\mathrm{F}(1,53)=15.4, p<0.001)$, and faster reaction times $(\mathrm{F}(1,53)=20.7, p<0.001)$ compared with WT mice
(Table 3). No group differences or interaction with genotype were observed, neither was there an effect of genotype on total trials completed ( $\mathrm{F}<1$, NS; Supplementary Table 2).

\section{Win-Stay/Lose-Shift Strategies of DAT KD and WT Mice}

Although both WT and KD tended to make more safe-stays over time $(\mathrm{F}(2,106)=3.0, p=0.055$; Figure $4 \mathrm{~b})$, a trial period $\times$ genotype $(\mathrm{F}(2,106)=4.2, p<0.05)$ and trial period $\times$ group $(\mathrm{F}(4,106)=8.8, p<0.001)$ interaction indicated that among good performing mice, $\mathrm{KD}$ mice exhibited less safe-stays than WT mice during trial period $3(p<0.01)$. No other differences in genotype were observed for any of the measures (Figure 4c, Supplementary Figure 5A and B).

\section{IGT Performance of Mice Treated with the Acute DAT Inhibitor GBR12909}

Overall, a trial period $\times$ group $(\mathrm{F}(4,152)=40.0, p<0.001)$ and trial period $\times$ GBR12909 $(\mathrm{F}(4,152)=3.2, p<0.05)$ interactions indicated similar overall group differences as above. 
a

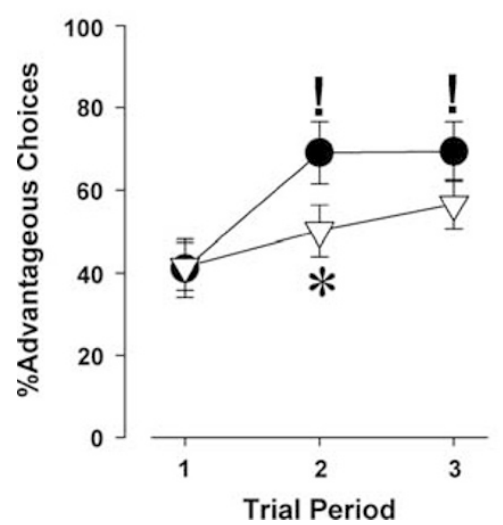

b

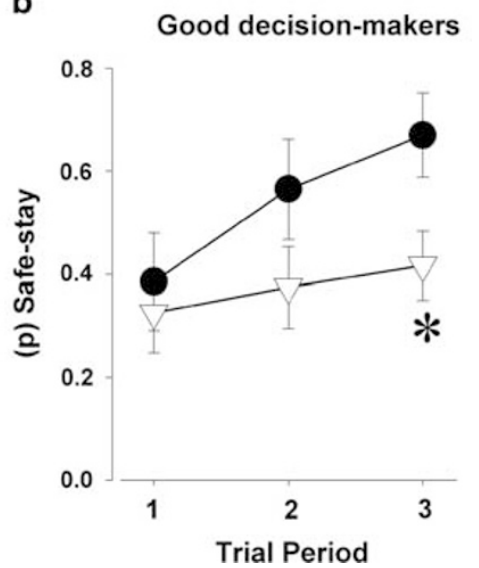

C

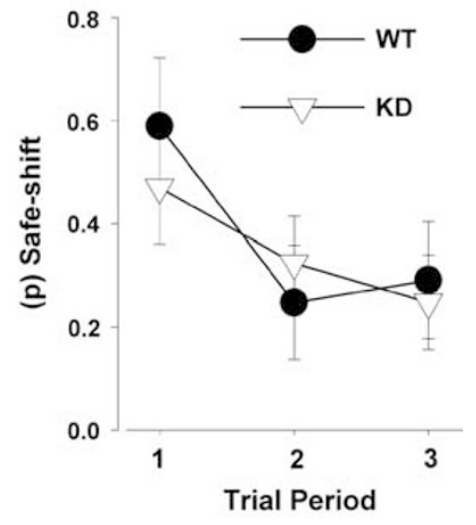

Figure 4 lowa Gambling Task (IGT) performance of dopamine transporter (DAT) knockdown (KD) and wild-type (WT) littermates identified as good decision makers. (a) WT mice rapidly increased advantageous choices over time, whereas KD mice increased more gradually and performed poorer in trial period 2. (b) Over time, both WT and KD mice made more low-reward safe-stays, although KD mice stayed significantly less compared with WT mice by the final trial period. (c) Over time, both WT and KD mice shifted less from the advantageous options after punishment (safe-shifts). Data are presented as the mean \pm SEM, ${ }^{*} p<0.05$ and ${ }^{\#} p<0.1$ when compared with WT, ! $p<0.05$ when compared with chance.

a

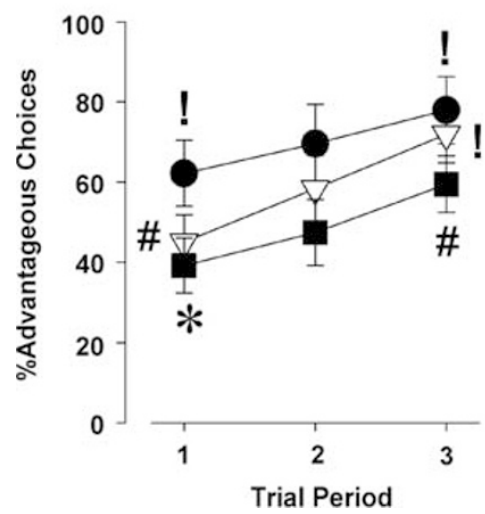

b

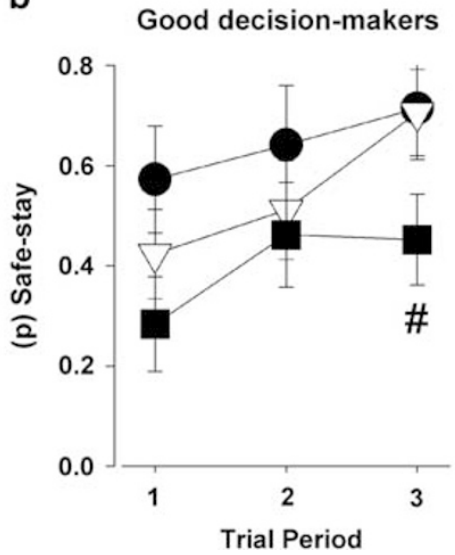

C

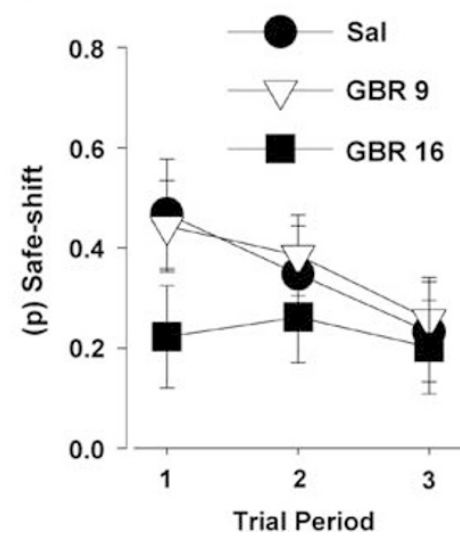

Figure 5 lowa Gambling Task (IGT) performance of C57BL/6 mice, identified as good decision makers, treated with the acute dopamine transporter (DAT) inhibitor GBRI2909. (a) Both saline-treated (Sal) mice and GBRI2909-treated (GBR 9 and GBR I6) mice increased advantageous choices over time. However, GBRI2909-treated mice exhibited impaired decision making compared with saline-treated mice, especially at $16 \mathrm{mg} / \mathrm{kg}$. (b) Over time, all mice made more low-reward safe-stays, although mice receiving GBRI 2909 at 16 mg/kg stayed significantly less compared with saline-treated mice. (c) No effect of GBR 2909 treatment was observed on the animals' decrease in shifting from the advantageous options after punishment (safe-shifts) over time. Data are presented as the mean \pm SEM, ${ }^{*} p<0.05$ and ${ }^{\#} p<0.1$ when compared with saline, ! $p<0.05$ when compared with chance.

Good decision makers. Although both saline- and GBR12909-treated mice learned to select advantageous choices over time $(\mathrm{F}(2,48)=32.1, p<0.001)$, mice receiving GBR12909 at $16 \mathrm{mg} / \mathrm{kg}$ made significantly less advantageous choices than saline-treated mice overall $(p<0.05$; effect size $[d]=0.95$; Figure $5 \mathrm{a})$. Moreover, saline-treated mice performed above chance in trial periods 1 and $3(p<0.05)$, whereas mice receiving GBR12909 at $16 \mathrm{mg} / \mathrm{kg}$ did not differ from chance and mice receiving $9 \mathrm{mg} / \mathrm{kg}$ GBR12909 were only above chance in trial period $3(p<0.05)$. No differences in treatment were observed in both the intermediate and poor decision makers (Supplementary Figure 6A and B). These findings indicate deleterious effects of GBR12909 on risk-based decision-making of mice.
Other behavioral measures. No overall effects of GBR12909, group differences, or interaction were observed, although a main effect of GBR12909 in the good decision makers $(\mathrm{F}(2,24)=8.1, p<0.01)$ indicated that mice receiving $9 \mathrm{mg} / \mathrm{kg}$ GBR12909 exhibited fewer omissions compared with saline-treated mice $(p<0.05$; Table 3$)$. There was no effect of GBR12909 on total trials completed (F=1.3, NS; Supplementary Table 2).

\section{Win-Stay/Lose-Shift Strategies of GBR12909-Treated Mice}

Although all good decision makers made more safe-stays over time $(\mathrm{F}(2,46)=8.6, p<0.01)$, mice receiving GBR12909 
at $16 \mathrm{mg} / \mathrm{kg}$ exhibited less safe-stays than saline-treated mice overall ( $p<0.05$; Figure 5b). Except for mice treated with $9 \mathrm{mg} / \mathrm{kg}$ GBR12909 making more risky-stays compared with saline-treated mice initially (Supplementary Figure 7A), no other differences were observed for any other measures (Figure 5c, Supplementary Figure 7B).

\section{DISCUSSION}

Impaired IGT performance was observed in $\mathrm{BD}$ patients compared with healthy subjects. In the single-session IGT task for mice, we demonstrated that dynamic decision making could be assessed when the animals were exposed to risk and reward contingencies similar to those in the human IGT. Stable subgroups were identified among mice, consistent with rats (Rivalan et al, 2009) and inter-individual differences in humans (Weller et al, 2010; illustrated in Figure 2e). Importantly, good decision makers increased safe-stays and decreased risky-stays over time, a pattern consistent with human IGT studies (Bechara et al, 2002b). As hypothesized, both chronic and acute reduced DAT functioning via genetic KD and GBR12909, respectively, deleteriously affected IGT performance in good decision makers. In mice, as in BD patients, this deficit was driven by a reduced likelihood to maintain responding for low rewards, without a difference for high rewards. Hence, the impaired decision-making profile of these mice was consistent with that of BD patients (Adida et al, 2011; Ibanez et al, 2012).

The IGT performance deficits of $\mathrm{BD}$ patients are consistent with previous studies (Adida et al, 2008; Clark et al, 2001; Yechiam et al, 2008), with deficits observed across several phases of BD (euthymia, depression, and mania; Adida et al, 2011). We provided novel analyses to investigate the underpinnings of this deficit. Manic BD patients studied here tended to pick less from the safe decks repeatedly (less safe-stays) and were more likely to switch to the risky decks after punishment (more safe-shifts). Such attendance to gains rather than loss are also seen in $\mathrm{BD}$ euthymic patients administered the $\mathrm{DA} \mathrm{D}_{2} / \mathrm{D}_{3}$ receptor agonist pramipexole (Burdick et al, 2014). In rats, DA $D_{1}$ and $\mathrm{D}_{2}$ receptor contributions to win-stay/lose-shift strategies have been assessed during reward unpredictability (St Onge et al, 2011). The lack of punishment or varied reward levels in this reward probability task limits direct comparison to these data, but highlights how this mouse IGT paradigm can be used to examine the neural contribution to such reduced safe-stays and increased safe-shifts. Understanding differences in punishment- and rewardrelated learning is critical given that depressed subjects make punishment-sensitive decisions in the IGT (Adida et al, 2011), whereas substance-dependent (Bechara et al, 2002b) and remitted BD subjects (Brambilla et al, 2012) attend to high rewards irrespective of risk.

This preference for high reward of $\mathrm{BD}$ patients and the development of the single-session mouse IGT enabled our primary investigation, determining whether this pattern is recapitulated in hyperdopaminergic mice because of reduced functional DAT levels. We observed that among regular C57BL/6J mice, some mice developed a preference for low-reward advantageous options ('good decision makers'). In contrast, other mice developed a bias for high-reward disadvantageous options ('poor decision makers'), whereas a third group of mice did not develop a response preference ('intermediate decision makers'). Such inter-individual differences have been observed in rat (Rivalan et al, 2009) and human IGT studies (Figure 2e), reflecting underlying risk-prone traits. Greater proportions of humans exhibit preference for advantageous choices (Bechara and Damasio, 2002a; Weller et al, 2010). The high number of poor or intermediate decision-making mice may represent too many risk-prone animals masking any genotypic or pharmacological effects, hence these differences were observed only in good decision makers. Despite subgroup differences in decision making over time, these groups did not differ on secondary measures of omissions, premature responses, and reaction times. This dissociation emphasizes the selectivity of risk-learning performance suggesting that it is unrelated to motor impulsivity or motivational features. Together, these data support the use of the single-session rodent IGT to examine risk-based decision making and highlight the importance of interindividual differences in risk preference.

Both chronic and acute reductions of functional DAT in mice negatively affected risky decision making. Within the good decision makers, KD mice exhibited impaired decision making compared with WT mice, similar to impaired IGT performance of BD patients compared with HCs. Similar risk preference of KD mice has been observed before in a multiple session rodent IGTs (Young et al, 2011), but importantly the present study utilizes a single-session IGT, consistent with the human IGT. Because of the similarity between tasks, we could analyze dynamic decision making after rewards or punishment as was conducted in humans. Poorer KD performance was likely mediated by their sensitivity to high reward, reflected by less likelihood of staying with low-reward options. The effects of acute DAT inhibition via GBR12909 treatment on decision making were similar to performance in KD mice. GBR12909 (16 mg/kg) significantly increased risk preference compared with saline driven by reduced tendency to stay at low rewards. Supporting these effects, we previously observed that GBR12909 modestly increased risk preference in a multiple session mouse IGT (van Enkhuizen et al, 2013b). Utilizing the same multiple session tasks in rats, however, simultaneous administration of GBR12909 and the norepinephrine transporter (NET) inhibitor atomoxetine was required in order to disrupt decision making (Baarendse et al, 2013). This disparity in results may reflect task differences or species differences because potencies of DAT and NET inhibitors vary between rats compared with mice and humans (Han and Gu, 2006; Rothman and Baumann, 2003). In humans, limited pharmacological IGT studies have been performed, although reduced DA activity impaired decision making in one study (Sevy et al, 2006). Interestingly, treatment with modafinil (an atypical stimulant with DAT inhibition properties) also impaired decision making in low pathological gamblers in the IGT (Zack and Poulos, 2009). Overall, the findings of reduced DAT functioning in mice are consistent with $\mathrm{BD}$ patients making less repeated picks from low-reward decks.

The importance of our findings is highlighted from studies indicating that polymorphisms in the DAT gene may 
induce lower DAT levels in patients with BD (Anand et al, 2011; Pinsonneault et al, 2011). Lower striatal DAT levels have also been observed in people with ADHD (Fusar-Poli et al, 2012) and seasonal affective disorder (Neumeister et al, 2001). People with ADHD also exhibit increased risky choices in the IGT (Mantyla et al, 2012), albeit to a lesser extent when compared with BD (Ibanez et al, 2012). Although impaired decision making of the reduced DAT model animals here could therefore resemble other clinical populations, the consistencies of BD patient IGT performance including the sensitivity for rewards, to our reduced DAT functioning in mice are striking. These findings are reinforced by previous observations of parallels between behavior of these DAT models and that of BD patients in other paradigms (Henry et al, 2013; Perry et al, 2009; van Enkhuizen et al, 2012). Moreover, supporting the increased reward-seeking trait of DAT KD mice, chronic DAT reduction also resulted in faster reaction times, fewer omissions, and increased premature responses in both the present and previous studies (Young et al, 2011). Similarly, and as observed previously (van Enkhuizen et al, 2013b), GBR12909 at $9 \mathrm{mg} / \mathrm{kg}$ reduced omissions, although no significant effects were found on motor impulsivity in contrast to the increased motor impulsivity with this dose that has been observed repeatedly in mice (Loos et al, 2010; van Enkhuizen et al, 2013b) and rats (Baarendse et al, 2013). Indeed, increased motivation and motor impulsivity of both DAT KD and to a lesser degree GBR12909-treated mice may be interpreted as consistent with the exaggerated hedonialike symptomatology observed in BD (Cassidy et al, 1998). Therefore, both DAT model animals resemble patients with $\mathrm{BD}$ in both behavior and putative etiology.

The differences on motivational and impulsivity measures seen between the chronic and acute DAT inhibition models may not be surprising. Previously, acute DAT blockade with GBR12909 did not affect reaction-times in mice (Loos et al, 2010; van Enkhuizen et al, 2013b). In contrast, constitutive DAT KD mice may have altered neurotransmission besides hyperdopaminergia (eg, DAT KD mice exhibit reduced choline transporter expression; Parikh et al, 2006), putatively contributing to these behavioral differences. Developmental changes resulting from reduced DAT expression may reproduce altered receptor levels in patients with $\mathrm{BD}$. Hence, chronic reductions of DAT expression in mice may model more aspects of $\mathrm{BD}$ than acute DAT inhibition alone. The etiological validity of the chronic DAT KD model is likely limited, however, by the fact that these animals express only $10 \%$ of the transporter (Zhuang et al, 2001), whereas only a $\sim 20 \%$ reduction of DAT availability is observed in euthymic BD patients (Anand et al, 2011). As yet, DAT levels in BD patients in the manic phase have yet to be established.

A limitation of the current study is the lack of DAT expression levels of our BD subjects performing the IGT. Hence, although we predicted increased risk-taking in mice with reduced DAT expression because of the reduced expression observed in unmedicated patients, it will be important for future studies to combine these behavioral assessments with physiological measurements, eg, via PET (Anand et al, 2011) or measurement in peripheral lymphocytes (Buttarelli et al, 2011). Another limitation is that experimental animals are kept in stable and controlled environments (ie, circadian rhythms/light exposure), whereas evidence suggests that environmental factors such as long day-lengths contribute to BD symptoms (Blumberg, 2012). Interestingly, long activity day-lengths may further induce a hyperdopaminergic state (Dulcis et al, 2013), which theoretically could exacerbate the reduced DAT levels of BD patients. Future tests will therefore include mice with $\sim 40-50 \%$ expression of the DAT and concurrent environmental manipulations such as aberrant light exposure to assess the relevance of these manipulations in modeling $\mathrm{BD}$ or other potentially DAT-mediated disorders such as ADHD. Cross-species translational studies can further help elucidate the differences in decision making and other measures between individuals with ADHD and BD. Examining a selective NET blocker may prove useful, given its role in the treatment of ADHD and previous implication in ameliorating prepulse inhibition deficits in DAT knockout mice (Arime et al, 2012; Yamashita et al, 2006). Future studies in humans and other model animals for BD (Einat, 2007; Roybal et al, 2007) will help delineate the mechanism(s) underlying impaired decision making and contribute to developing therapeutics aimed to treat these deficits.

In summary, BD patients exhibit impaired decision making in the IGT. Using post-reward/punishment decisionmaking measurements developed from animal studies, we identified evidence to support a high-reward sensitivity in these patients. The development of a dynamic single-session mouse IGT, wherein mice increase advantageous choices over time and post-reward/punishment measurements can be examined aids our translational work. Chronic and acute reductions of DAT functioning in mice deleteriously impacted risk-based decision-making, making mice sensitive to high rewards and mimicking deficits of $\mathrm{BD}$ patients. DAT reductions may therefore contribute to poor decision making under risk in BD patients. Finally, the single-session IGT may be used to assess decision-making deficits in other animal models of psychiatric disorders and test putative treatments.

\section{FUNDING AND DISCLOSURE}

Dr Geyer has received research grant support from NIH, the US Veteran's Administration VISN 22 Mental Illness, Research, Education, and Clinical Center, Intracellular Therapeutics, and Johnson \& Johnson. He has also received consulting compensation from Abbott, Cerca, Dart Neuroscience, Merck, Omeros, Takeda, and Teva, and holds an equity interest in San Diego Instruments. Dr Young has received research support from the NIH, the US Veteran's Administration VISN 22 Mental Illness, Research, Education, and Clinical Center, Cerca Insights, Lundbeck Ltd, and Omeros. He has also received consulting compensation from Amgen. The funding support did not have any role in the design, collection, or analysis of studies. The remaining authors declare no conflict of interest.

\section{ACKNOWLEDGEMENTS}

We thank Drs Berend Olivier, Catharine Winstanley, Ms Mahalah Buell, and Mr Richard Sharp for their support. These studies were supported by NIH grants R01-MH071916, 
R01-MH042228, and the Veteran's Administration VISN 22 Mental Illness Research, Education, and Clinical Center.

\section{REFERENCES}

Adida M, Clark L, Pomietto P, Kaladjian A, Besnier N, Azorin JM et al (2008). Lack of insight may predict impaired decision making in manic patients. Bipolar disorders 10: 829-837.

Adida M, Jollant F, Clark L, Besnier N, Guillaume S, Kaladjian A et al (2011). Trait-related decision-making impairment in the three phases of bipolar disorder. Biol Psychiatry 70: 357-365.

Anand A, Barkay G, Dzemidzic M, Albrecht D, Karne H, Zheng QH et al (2011). Striatal dopamine transporter availability in unmedicated bipolar disorder. Bipolar Disorder 13: 406-413.

Arime Y, Kasahara Y, Hall FS, Uhl GR, Sora I (2012). Corticosubcortical neuromodulation involved in the amelioration of prepulse inhibition deficits in dopamine transporter knockout mice. Neuropsychopharmacology 37: 2522-2530.

Baarendse PJ, Winstanley CA, Vanderschuren LJ (2013). Simultaneous blockade of dopamine and noradrenaline reuptake promotes disadvantageous decision making in a rat gambling task. Psychopharmacology (Berl) 225: 719-731.

Bechara A, Damasio AR, Damasio H, Anderson SW (1994). Insensitivity to future consequences following damage to human prefrontal cortex. Cognition 50: 7-15.

Bechara A, Damasio H (2002a). Decision-making and addiction (part I): impaired activation of somatic states in substance dependent individuals when pondering decisions with negative future consequences. Neuropsychologia 40: 1675-1689.

Bechara A, Dolan S, Hindes A (2002b). Decision-making and addiction (part II): myopia for the future or hypersensitivity to reward? Neuropsychologia 40: 1690-1705.

Blumberg HP (2012). Euthymia, depression, and mania: what do we know about the switch? Biol Psychiatry 71: 570-571.

Brambilla P, Perlini C, Bellani M, Tomelleri L, Ferro A, Cerruti S et al (2012). Increased salience of gains versus decreased associative learning differentiate bipolar disorder from schizophrenia during incentive decision making. Psychol Med 43: 1-10.

Burdick KE, Braga RJ, Gopin CB, Malhotra AK (2014). Dopaminergic influences on emotional decision making in euthymic bipolar patients. Neuropsychopharmacology 39: 274-282.

Buttarelli FR, Fanciulli A, Pellicano C, Pontieri FE (2011). The dopaminergic system in peripheral blood lymphocytes: from physiology to pharmacology and potential applications to neuropsychiatric disorders. Curr Neuropharmacol 9: 278-288.

Cassidy F, Forest K, Murry E, Carroll BJ (1998). A factor analysis of the signs and symptoms of mania. Arch Gen Psychiatry 55: 27-32.

Christodoulou T, Lewis M, Ploubidis GB, Frangou S (2006). The relationship of impulsivity to response inhibition and decisionmaking in remitted patients with bipolar disorder. Eur Psychiatry 21: 270-273.

Clark L, Iversen SD, Goodwin GM (2001). A neuropsychological investigation of prefrontal cortex involvement in acute mania. Am J Psychiatry 158: 1605-1611.

de Visser L, Homberg JR, Mitsogiannis M, Zeeb FD, Rivalan M, Fitoussi A et al (2011). Rodent versions of the Iowa Gambling Task: opportunities and challenges for the understanding of decision-making. Front Neurosci 5: 109.

Dulcis D, Jamshidi P, Leutgeb S, Spitzer NC (2013). Neurotransmitter switching in the adult brain regulates behavior. Science 340: 449-453.

Dunn LM, Dunn LM (1997). Peabody Picture Vocabulary Test. 3rd edn. Pearson Assessments: Bloomington, MN.

Einat H (2007). Different behaviors and different strains: potential new ways to model bipolar disorder. Neurosci Biobehav Rev 31: 850-857.
Floresco SB St, Onge JR, Ghods-Sharifi S, Winstanley CA (2008). Cortico-limbic-striatal circuits subserving different forms of costbenefit decision making. Cogn Affect Behav Neurosci 8: 375-389.

Fond G, Bayard S, Capdevielle D, Del-Monte J, Mimoun N, Macgregor A et al (2013). A further evaluation of decisionmaking under risk and under ambiguity in schizophrenia. Eur Arch Psychiatry Clin Neurosci 263: 249-257.

Fusar-Poli P, Rubia K, Rossi G, Sartori G, Balottin U (2012). Striatal dopamine transporter alterations in ADHD: pathophysiology or adaptation to psychostimulants? A meta-analysis. Am J Psychiatry 169: 264-272.

Greenwood TA, Schork NJ, Eskin E, Kelsoe JR (2006). Identification of additional variants within the human dopamine transporter gene provides further evidence for an association with bipolar disorder in two independent samples. Mol Psychiatry 11: 125-133.

Han DD, Gu HH (2006). Comparison of the monoamine transporters from human and mouse in their sensitivities to psychostimulant drugs. BMC Pharmacol 6: 6.

Henry BL, Minassian A, Patt VM, Hua J, Young JW, Geyer MA et al (2013). Inhibitory deficits in euthymic bipolar disorder patients assessed in the human behavioral pattern monitor. J Affect Disord 150: 948-954.

Horschitz S, Hummerich R, Lau T, Rietschel M, Schloss P (2005). A dopamine transporter mutation associated with bipolar affective disorder causes inhibition of transporter cell surface expression. Mol Psychiatry 10: 1104-1109.

Ibanez A, Cetkovich M, Petroni A, Urquina H, Baez S, GonzalezGadea ML et al (2012). The neural basis of decision-making and reward processing in adults with euthymic bipolar disorder or attention-deficit/hyperactivity disorder (ADHD). PLoS One 7: e37306.

Insel TR (2007). From animal models to model animals. Biol Psychiatry 62: 1337-1339.

Jollant F, Guillaume S, Jaussent I, Bellivier F, Leboyer M, Castelnau D et al (2007). Psychiatric diagnoses and personality traits associated with disadvantageous decision-making. Eur Psychiatry 22: 455-461.

Lin CH, Chiu YC, Cheng CM, Hsieh JC (2008). Brain maps of Iowa gambling task. BMC Neurosci 9: 72.

Loos M, Staal J, Schoffelmeer AN, Smit AB, Spijker S, Pattij T (2010). Inhibitory control and response latency differences between C57BL/6J and DBA/2J mice in a Go/No-Go and 5 -choice serial reaction time task and strain-specific responsivity to amphetamine. Behav Brain Res 214: 216-224.

Manji HK, Quiroz JA, Payne JL, Singh J, Lopes BP, Viegas JS et al (2003). The underlying neurobiology of bipolar disorder. World Psychiatry 2: 136-146.

Mantyla T, Still J, Gullberg S, Del Missier F (2012). Decision making in adults with ADHD. J Atten Disord 16: 164-173.

Must A, Horvath S, Nemeth VL, Janka Z (2013). The Iowa Gambling Task in depression-what have we learned about sub-optimal decision-making strategies? Front Psychol 4: 732.

Neumeister A, Willeit M, Praschak-Rieder N, Asenbaum S, Stastny J, Hilger $\mathrm{E}$ et al (2001). Dopamine transporter availability in symptomatic depressed patients with seasonal affective disorder and healthy controls. Psychol Med 31: 1467-1473.

Parikh V, Apparsundaram S, Kozak R, Richards JB, Sarter M (2006). Reduced expression and capacity of the striatal highaffinity choline transporter in hyperdopaminergic mice. Neuroscience 141: 379-389.

Perry W, Minassian A, Paulus MP, Young JW, Kincaid MJ, Ferguson EJ et al (2009). A reverse-translational study of dysfunctional exploration in psychiatric disorders: from mice to men. Arch Gen Psychiatry 66: 1072-1080.

Pinsonneault JK, Han DD, Burdick KE, Kataki M, Bertolino A, Malhotra AK et al (2011). Dopamine transporter gene variant affecting expression in human brain is associated with bipolar disorder. Neuropsychopharmacology 36: 1644-1655. 
Rao JS, Kellom M, Reese EA, Rapoport SI, Kim HW (2012). Dysregulated glutamate and dopamine transporters in postmortem frontal cortex from bipolar and schizophrenic patients. J Affect Disord 136: 63-71.

Rivalan M, Ahmed SH, Dellu-Hagedorn F (2009). Risk-prone individuals prefer the wrong options on a rat version of the Iowa Gambling Task. Biol Psychiatry 66: 743-749.

Rivalan M, Coutureau E, Fitoussi A, Dellu-Hagedorn F (2011). Inter-individual decision-making differences in the effects of cingulate, orbitofrontal, and prelimbic cortex lesions in a rat gambling task. Front Behav Neurosci 5: 22.

Rothman RB, Baumann MH (2003). Monoamine transporters and psychostimulant drugs. Eur J Pharmacol 479: 23-40.

Roybal K, Theobold D, Graham A, DiNieri JA, Russo SJ, Krishnan V et al (2007). Mania-like behavior induced by disruption of CLOCK. Proc Natl Acad Sci USA 104: 6406-6411.

Sevy S, Hassoun Y, Bechara A, Yechiam E, Napolitano B, Burdick K et al (2006). Emotion-based decision-making in healthy subjects: short-term effects of reducing dopamine levels. Psychopharmacology (Berl) 188: 228-235.

St Onge JR, Abhari H, Floresco SB (2011). Dissociable contributions by prefrontal D1 and D2 receptors to risk-based decision making. J Neurosci 31: 8625-8633.

St Onge JR, Floresco SB (2009). Dopaminergic modulation of riskbased decision making. Neuropsychopharmacology 34: 681-697.

Tohen M, Chengappa KN, Suppes T, Zarate CA Jr, Calabrese JR, Bowden CL et al (2002). Efficacy of olanzapine in combination with valproate or lithium in the treatment of mania in patients partially nonresponsive to valproate or lithium monotherapy. Arch Gen Psychiatry 59: 62-69.

van Enkhuizen J, Geyer MA, Kooistra K, Young JW (2012). Chronic valproate attenuates some, but not all, facets of manialike behaviour in mice. The international journal of neuropsychopharmacology/official scientific journal of the Collegium Internationale Neuropsychopharmacologicum 16: 1-11.

van Enkhuizen J, Geyer MA, Kooistra K, Young JW (2013a). Chronic valproate attenuates some, but not all, facets of mania-like behaviour in mice. Int J Neuropsychopharmacol 16: 1021-1031.

van Enkhuizen J, Geyer MA, Young JW (2013b). Differential effects of dopamine transporter inhibitors in the rodent Iowa gambling task: Relevance to mania. Psychopharmacology (Berl) 225: 661-674.
Vawter MP, Freed WJ, Kleinman JE (2000). Neuropathology of bipolar disorder. Biol Psychiatry 48: 486-504.

Weller JA, Levin IP, Bechara A (2010). Do individual differences in Iowa Gambling Task performance predict adaptive decision making for risky gains and losses? J Clin Exp Neuropsychol 32: 141-150.

Yamashita M, Fukushima S, Shen HW, Hall FS, Uhl GR, Numachi Y et al (2006). Norepinephrine transporter blockade can normalize the prepulse inhibition deficits found in dopamine transporter knockout mice. Neuropsychopharmacology 31: 2132-2139.

Yechiam E, Hayden EP, Bodkins M, O'Donnell BF, Hetrick WP (2008). Decision making in bipolar disorder: a cognitive modeling approach. Psychiatry Res 161: 142-152.

Young JW, Goey AK, Minassian A, Perry W, Paulus MP, Geyer MA (2010). GBR 12909 administration as a mouse model of bipolar disorder mania: mimicking quantitative assessment of manic behavior. Psychopharmacology (Berl) 208: 443-454.

Young JW, van Enkhuizen J, Winstanley CA, Geyer MA (2011). Increased risk-taking behavior in dopamine transporter knockdown mice: further support for a mouse model of mania. J Psychopharmacol (Oxf) 25: 934-943.

Zack M, Poulos CX (2009). Effects of the atypical stimulant modafinil on a brief gambling episode in pathological gamblers with high $v s$ low impulsivity. J Psychopharmacol 23: 660-671.

Zeeb FD, Robbins TW, Winstanley CA (2009). Serotonergic and dopaminergic modulation of gambling behavior as assessed using a novel rat gambling task. Neuropsychopharmacology 34: 2329-2343.

Zeeb FD, Winstanley CA (2011). Lesions of the basolateral amygdala and orbitofrontal cortex differentially affect acquisition and performance of a rodent gambling task. J Neurosci 31: 2197-2204.

Zeeb FD, Wong AC, Winstanley CA (2013). Differential effects of environmental enrichment, social-housing, and isolation-rearing on a rat gambling task: dissociations between impulsive action and risky decision-making. Psychopharmacology (Berl) 225: 381-395.

Zhuang X, Oosting RS, Jones SR, Gainetdinov RR, Miller GW, Caron MG et al (2001). Hyperactivity and impaired response habituation in hyperdopaminergic mice. Proc Natl Acad Sci USA 98: 1982-1987.

Supplementary Information accompanies the paper on the Neuropsychopharmacology website (http://www.nature.com/npp) 